\title{
A practical depth-of-interaction PET/MR detector with dichotomous-orthogonal-symmetry decoding
}

\author{
Yuxuan Zhang, Hossain Baghaei, Han Yan, Wai-Hoi Wong* \\ From PSMR 2015: 4th Conference on PET/MR and SPECT/MR \\ La Biodola, Isola d'Elba, Italy. 17-21 May 2015
}

University of Texas MD Anderson Cancer Center, Houston, Texas, USA
Conventional dual-end depth-of-interaction (DOI) PET detector readout requires two 2D SiPM arrays; with top and bottom SiPM reading the same pixel, there is information redundancy. We proposed a dichotomous-orthogonal-symmetric (DOS) dual-end DOI readout to eliminate this redundancy to significantly reduce SiPM usage, electronic channels, and heat load. Reflecting films are used within the scintillator array to channel light exiting the top along the X-direction, while light exiting the bottom is channeled along the orthogonal Y-direction. Despite the unidirectional channeling on each end, the top readout can provide $\mathrm{X}-\mathrm{Y}$ information using two 1-D SiPM arrays; similarly, the bottom readout also provides X-Y information with two 1-D SiPM arrays. Thus four 1-D SiPM arrays $(4 \mathrm{xN})$ are used to decode XYZ to replace two 2D SiPM arrays $(2 \mathrm{NxN})$; SiPM usage is reduced from $2 \mathrm{~N}^{* *} 2$ to $4 \mathrm{~N}$. Monte Carlo simulations (GATE) were carried out to study the XY decoding accuracy, energy resolution, and DOI resolution. Coupling the DOS-DOI design with a channel-decoding scheme, an array of $15 \times 15$ LSO $(2.4 \times 2.4 \times 20 \mathrm{~mm}$ pixels) can be decoded by $18 \mathrm{SiPMs}$ (2 rows of nine $3 \times 3 \mathrm{~mm}$ SiPM) on top and $18 \mathrm{SiPMs}$ at bottom, thus achieving a 10X reduction in SiPM usage, electronic channels and heat load. For BGO detectors, an $8 \times 8$ array $(2.4 \times 2.4 \times 20 \mathrm{~mm}$ pixels $)$ can be achieved with $6.4 \mathrm{X}$ reduction. Simulations show 5-6mm DOI resolution, $0.45-0.96 \mathrm{~mm} \mathrm{XY}$ decoding blurring, 20-24\% energy resolution. This study shows the feasibility of the DOS-DOI design. Even comparing to non-DOI detectors, there is a $5 \mathrm{X} / 3 \mathrm{X} \mathrm{SiPM}$ reduction for $\mathrm{LSO} / \mathrm{BGO}$. The proposed detector may yield practical ultrahigh-resolution PET/MR systems with depth-of-interaction with a production cost below current non-DOI systems.

Published: 18 May 2015

doi:10.1186/2197-7364-2-S1-A12

Cite this article as: Zhang et al:: A practical depth-of-interaction PET/MR detector with dichotomous-orthogonalsymmetry decoding. EJNMMI Physics 2015 2(Suppl 1):A12.

\section{SpringerOpen ${ }^{\circ}$}

(C) 2015 Zhang et al; licensee Springer. This is an Open Access article distributed under the terms of the Creative Commons Attribution License (http://creativecommons.org/licenses/by/4.0), which permits unrestricted use, distribution, and reproduction in any medium, provided the original work is properly cited. 